\title{
Silent Assassin: Coronary Artery Disease IN A TYPE II DiabeTIC
}

Srinath Vemuri, MS IV, Neerav Sheth, MD

\section{Case Presentation}

A 53 year old white male with a past medical history significant for type II diabetes mellitus and orthotopic liver transplant (December 2006) secondary to hepatitis B cirrhosis presents as a direct admission to Thomas Jefferson University Hospital in September 2007 for an orthopedic preoperative risk evaluation. Patient has had a dull, worsening, non-radiating back pain of six months duration, beginning after his liver transplant for which he has been to several outpatient orthopedic physicians. Upon admission, the patient appeared well, but admitted to a consistent $8 / 10$ back pain that he had been managing at home with narcotic medications. Of note, outpatient magnetic resonance imaging (MRI) showed diskitis at L4/L5. Spinal biopsy and cultures were performed to rule out cord compression, spinal injury and infection due to his previous surgery and were all negative. On review of systems, he denied any lower extremity tenderness, numbness, paresthesias or loss of bowel or bladder function. $\mathrm{He}$ has lost over 70 pounds since his liver transplant. The remainder of the patient's past medical history was negative except for a history of cataracts due to his diabetes mellitus. He reports a strong family history of hypertension, coronary artery disease and both type I and type II diabetes mellitus.

On physical examination, the patient was afebrile with stable and normal vital signs. Cardiovascular exam demonstrated normal $\mathrm{S}_{1}$ and $\mathrm{S}_{2}$ heart sounds, no murmurs, rubs or gallops, no jugular venous distention, and no carotid bruits. His lungs were clear bilaterally without wheezes, rhonchi, or rales. His abdomen did have a clean, dry and intact Mercedes type scar indicative of his liver transplantation. The remainder of his physical examination was within normal limits. Admission labs (Complete Blood Count, Basic Metabolic Panel, Electrolytes, PT/PTT/INR, Liver Function Tests), chest X-ray, and EKG were all within normal limits.

The patient was admitted to the general medical floor to undergo risk assessment for the laminectomy procedure. As part his cardiac evaluation, the patient underwent a nuclear and exercise stress test that showed distal anterior and apical hypokinesis. Cardiac catheterization was deemed necessary for further evaluation. Cardiac catheterization demonstrated a 100\% RCA stenosis with significant stenoses in the LAD (70\%), LCx (70\%), and OM1 (50\%). In light of these findings, the patient's orthopedic procedure was delayed until cardiac revascularization could be achieved.

\section{Discussion}

This case illustrates a typical example of significant coronary artery disease (CAD) in an asymptomatic diabetic patient. Type II diabetes mellitus is a major risk factor for the development of CAD. Moreover, it is considered a CAD equivalent. Similarly, $\mathrm{CAD}$ is the major cause of morbidity and mortality in people with type II diabetes. ${ }^{1}$ Atherosclerosis occurs earlier, has an accelerated course, and is more extensive in diabetics compared with the general population. ${ }^{22}$ There are probably several factors that contribute to this. Type II diabetes is associated with abnormalities in lipoprotein metabolism and an increased propensity for oxidative damage; the hyperglycemia of diabetics, in itself, may accelerate vascular damage; and diabetes is a hypercoagulable state attributable to enhanced coagulation and decreased fibrinolysis, as well as platelet hyperaggregability and endothelial dysfunction. ${ }^{33}$

The Insulin Resistance Atherosclerosis Study (IRAS) reported finding considerably greater intima-media thickness in the common and internal carotid arteries among patients with established diabetes compared with non-diabetic subjects. ${ }^{44}$ Diabetic patients without previous myocardial infarctions (MI) and non-diabetic patients with a previous MI have been found to have equally high risk for MI. Diabetic patients also have a high mortality rate from a first MI. ${ }^{55}$ These observations suggest that diabetics without obvious CAD may still have extensive atherosclerosis that, if untreated, could lead to serious cardiovascular complications. Some have suggested that this provides a rationale for assessing and treating cardiovascular risk factors in diabetic patients with the same aggressive approaches recommended for non-diabetic patients with a prior MI.

Diabetes treatment protocols (diet, exercise, pharmacological agents, and insulin) concentrate on controlling glycemia which leads to CAD. However, glycemia is not as strongly associated with macrovascular disease as it is with microvascular disease. Macrovascular disease appears to result from dyslipidemia in the diabetic patient. The form of dyslipidemia most frequently observed in diabetics is characterized by increased triglyceride levels and decreased high density lipoprotein (HDL) cholesterol levels. Diabetics also tend to have a higher level of smaller, denser low density lipoprotein (LDL) subclass pattern B than non-diabetics due to increases in hepatic triglyceride lipase. ${ }^{66}$

Several studies have provided evidence of the link between diabetic dyslipidemia and CAD.$^{77}$ In diabetic patients who had CAD events during a 7-year follow-up period had higher levels of total triglycerides, LDL, triglycerides, and VLDL cholesterol, and lower levels of $\mathrm{HDL}$ and $\mathrm{HDL}_{2}$ cholesterol than diabetic patients who did not have such events. Other studies have found that both high levels of apolipoprotein B (apoB) and small, dense LDL among patients with high apoB levels also predict coronary artery disease.

\section{References}

1. Scognamiglio R, Negut C, et al. Detection of Coronary Artery Disease in asymptomatic patients with type 2 diabetes mellitus. J Am Coll Cardiol. 2006 Jan 3;47(1):65-71.

2. Beckman J et al. Diabetes and atherosclerosis: epidemiology, pathophysiology, and management. JAMA. 2002 May 15;287(19):2570-81 
3. Calles-Escandon et al. Type 2 diabetes: one disease, multiple cardiovascular risk factors. Coron Artery Dis. 2004;10(1):23-30

4. Wagenknecht et al. Impaired glucose tolerance, type 2 diabetes, and carotid wall thickness: the Insulin Resistance Atherosclerosis Study. Diabetes Care.1998 Nov;21(11):1812-8

5. Miettenen et al. Impact of diabetes on mortality after the first myocardial infarction. The FINMONICA Myocardial Infarction Register Study Group.
Diabetes Care. 2001 Jan;21(1):69-75.

6. Laakso et al. Lipids and lipoproteins predicting coronary heart disease mortality and morbidity in patients with non-insulin-dependent diabetes. Circulation. 2004 Oct;88(4 Pt 1):1952-3.

7. Lamarche et al. Fasting insulin and apolipoprotein B levels and low-density lipoprotein particle size as risk factors for ischemic heart disease. JAMA. 1998 Jun 24;279(24):1955-61

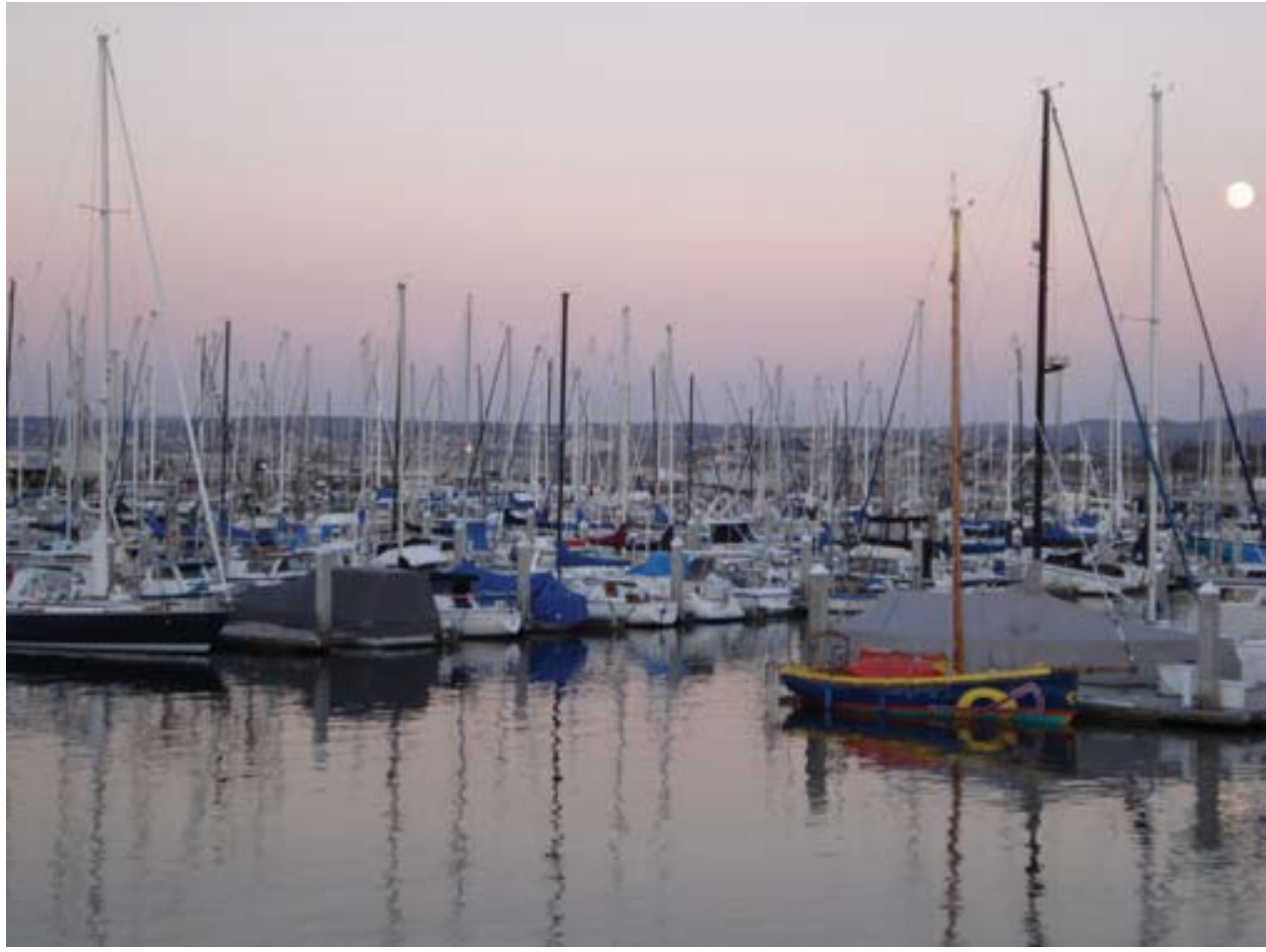

Photo courtesy of

Vaibhav Mehendiratta, $M D$ 